\title{
Catalogue of HI maps of galaxies
}

\section{Analysis of the data ${ }^{\star}$}

\section{M.C. Martín}

Instituto Argentino de Radioastronomía (IAR), Casilla de Correo 5 Villa Elisa (1894), Provincia de Bs. As., Argentina

Received August 1, 1995; accepted December 11, 1997

\begin{abstract}
We use some of the maps of the catalogue presented in Paper I to provide some evidence for global conditions that must be fulfilled by the galaxies to have extended hydrogen. For this purpose, we tried to find possible connections between the HI gas extension and other properties of the galaxies (morphological type, surface brightness, gas density, etc.).

With isophotal hydrogen diameters of a large sample, we could observe that optically smaller galaxies seem to have greater relative $\mathrm{HI}$ extensions. By means of the relation with the apparent HI surface density, we found an expression that should provide a rough estimate of the gas extension.

With respect to the dependence on morphological type, we could not find any significant correlation either for the real HI surface density or the relative gas extension. Nevertheless, whereas for spiral and irregular galaxies the real HI surface density exhibits a broad range of values, the values are rather lower for elliptical and S0 galaxies.
\end{abstract}

Key words: galaxies: ISM; structure - radio lines: galaxies

\section{Introduction}

The outer galactic HI layer is without doubt an important tracer of the system kinematics and provides crucial evidence for the presence of dark matter in the outer parts of the galaxies. At present, the search for extensive emission of neutral hydrogen in the majority of galaxies shows that the gas does not spread farther than 2 or 3 Holmberg radii. This so for isolated galaxies, for systems in a group or galaxies in interaction (Hewitt et al. 1983; Briggs et al.

\footnotetext{
* Table 1 is also available in electronic form at the CDS via anonymous ftp 130.79 .128 .5 or via http://cdsweb.ustrasbg.fr/Abstract.html
}

1980). However, some galaxies have been found that exhibit very large HI envelopes, like DDO 154 (Krumm \& Burstein 1984), NGC 262 (Morris \& Wannier 1980), NGC 5236 (Bottinelli \& Gouguenheim 1973), NGC 4449 (Bajaja et al. 1994), etc. Some of these extensions may be present as envelopes, warps, tails or bridges. These features can easily be explained when the galaxy has a clear interaction with another one, but it is difficult when there are no neighbouring galaxies.

The search for relations between the gas extension and other properties of galaxies has not been fruitful as yet. Particularly, it is not clear if the ratio of HI to optical diameter of a galaxy depends on its morphological type. With respect to this dependence, the following results have been found by different authors: the relative gas extension shows a slight variation along the whole morphological sequence (Fouqué 1982, 1983); the dependence is only for spiral galaxies (Bottinelli 1971) or only for early-type galaxies (Balkowski et al. 1972; Balkowski 1979; Krumm \& Salpeter 1980); the relative gas extension does not depend at all on the morphological type (Krumm \& Salpeter 1979; Bosma 1981; Hewitt et al. 1983). Furthermore, it was found that irregular galaxies are frequently rich in neutral hydrogen gas and some of them have HI distributions extended well beyond their optical boundaries (Huchtmeier et al. 1981; Gallagher \& Hunter 1984).

To sum it up, different results so far have shown that the relative size of the gas does not appear to have a clear dependence on any physical properties of the galaxies, such as surface brightness, luminosity, etc. (Haynes et al. 1984; Sancisi 1987; Giovanelli \& Haynes 1988). The exception is for the morphology, although the dependence, if real, is very weak and difficult to discern, as discussed above.

With the aim to improve the relationships of these types, we analyse a large sample of galaxies. This will be useful to find the condition of large HI disks.

The sample selected for this work is analysed in Sect. 2 . The choice of the distance concerning the parameters used 
here, is discussed in Sect. 3. Finally the results obtained are presented in Sect. 4 and the conclusion in Sect. 5 .

\section{Analysis of the data}

When a galaxy is observed with low spatial resolution relative to the gas extension, the HI maps show a roughly gaussian distribution. When the spatial resolution grows, some structures appear, such as inner and external rings, several concentrations, etc. For high resolution observations, fine structures such as spiral arms, gas shells, "holes" in the HI distribution, etc., start to be resolved. In all cases, the observed distribution of the gas is the convolution of the real one with the antenna response.

We have collected the maps of the integral HI density distribution in the catalogue of Paper I. As the main parameter of the sample we are dealing with is the real extension of the gas, we ought to make the deconvolution. Then, among these maps, we kept those whose contour lines show no detailed structures which would complicate a further analysis. In this way, we can apply a simplified gaussian model for the gas distribution that masks particular details, asymmetries and some large-scale features that are common in most galaxies.

It is known that the real HI distribution often shows a central depression, especially in early spiral galaxies (Roberts 1975; Sersic 1980). It is convenient that the model representative of the gas distribution takes this fact into account. A frequently-used symmetrical model which gives a useful rough representation for most spiral galaxies is the sum of two gaussians (Shostak 1978; Hewitt et al. 1983). One gaussian distribution of HI gas may work for irregular galaxies, because of their flat gaseous disk distribution. Thus, we have adopted one and two gaussian models of the HI distribution for irregular and spiral galaxies, respectively, which are represented by the following expression:

$\sigma_{\mathrm{HI}}(r)=a_{1} \mathrm{e}^{\frac{-\left(r^{2} / \cos ^{2} i\right)}{\rho_{1}^{2}}}+a_{2} \mathrm{e}^{\frac{-\left(r^{2} / \cos ^{2} i\right)}{\rho_{2}^{2}}}$

where $r$ is the radial distance, $i$ is the inclination of the galaxy and $a_{1}, a_{2}, \rho_{1}$ and $\rho_{2}$ are model parameters, with $a_{2}=C_{1} a_{1} ; \rho_{2}=C_{2} \rho_{1}$.

For a one-gaussian model, $a_{2}=0\left(C_{1}=0\right)$. For a twogaussian model, Hewitt et al. (1983) adopt $C_{1}=-0.6$ for the relative strength of the gaussians, which produces a deeper central depression than the Shostak model (1978) with $C_{1}=-0.3$. We use $C_{2}=0.5$ for the relative width of the gaussians. The model parameter $a_{1}$ is concerned with the strength of the emission. We have normalised the intensity of emission to the central values on the maps. The $\rho_{1}$ parameter is the factor that radially compresses or expands the HI distribution, and is basically the parameter on which the fit depends.

In order to make the fit, we first determined the major axis of the gas distribution from the map. On this major axis, we obtained the radial distances $(r)$ and the observed HI surface density at these distances. It is worth to noting that the HI major axis may not be coincident with the optical axis. In fact, this issue lead us to consider only the papers with maps of the total distribution of the gas emission, and reject those papers with observations along only one axis of the galaxy.

The expression (1) was convolved with the beam width of the telescope used in the observation, which was supposed gaussian as well. This convolution must reproduce the distribution of the HI surface density observed in the map. Then, we iteratively vary $\rho_{1}$ in expression (1) until the best mean least square fit between the calculated and observed values is achieved. With respect to the relative strength of the gaussians, represented by the $C_{1}$ parameter, we took its values at -0.6 or -0.3 , depending on which gave the best fit to the observations. The result was that for galaxies with morphological type earlier than 4 , the number of objects that best fit with $C_{1}=-0.3$ is approximately the same as the one with $C_{1}=-0.6$. However, for later-type galaxies, the number of best fits with $C_{1}=-0.3$ is remarkably large. This result may be in agreement with the fact that the central depression in the HI distribution seems to be less pronounced in late-type systems, which possess small bulges.

For comparison with optical isophotal diameters, the best-fit model is used to compute the HI isophotal diameter, then corrected by beam and inclination effects. The isophotal diameters are defined according to a particular isophote. By inspection of the data, we find that the best sensitivity reached in the observations is, in most cases, $2.510^{19}$ at $\mathrm{cm}^{-2}$, and we have adopted this value for estimating the $\mathrm{HI}$ isophotal diameter $\left(D_{\mathrm{HI}}\right)$. We only kept those galaxies measured until a surface density less or equal to $1510^{19}$ at $\mathrm{cm}^{-2}$, because we have found that the extrapolation is not valid for larger values.

In Table 1, we have listed the galaxies that make up the sample that we use for the subsequent analysis. The optical parameters are extracted from the LEDA catalogue (Lyon-Meudon Extragalactic Database, first and second edition). First entries to the table are:

Column 1: Galaxy name.

Column 2: Alternative name of the galaxy.

Column 3: Optical isophotal diameter measured to the surface brightness level of $25 \mathrm{mg} /[]^{\prime \prime}$ corrected for galactic and internal absorption $\left(D_{0}\right)$, in arc minutes.

Column 4: Morphological type.

Column 5: Inclination, in degrees.

Column 6: Distance, in Mpc. When the distance is uncertain, the extreme assumed values are quoted. See following discussion.

Column 7: The linear diameter $A(0)$ in kpc, from Cols. 3 and 6.

Column 8: HI mass, $M_{\mathrm{HI}}$, in $10^{9} M_{0}$. The adopted values of $M_{\mathrm{HI}}$ are discussed in Sect. 4.

Column 9: Mean apparent surface density of $\mathrm{HI}, \rho_{\mathrm{HI}}$, in 
$10^{21}$ at $\mathrm{cm}^{-2}$, from Cols. 7 and 8.

Column 10: Mean real surface density, $\Sigma_{\mathrm{HI}}$, in $10^{20}$ at $\mathrm{cm}^{-2}$, from Col. 8 and the $\mathrm{HI}$ isophotal diameter, $D_{\mathrm{HI}}$ (see Col. 3 of second entry).

Second entries to the Table are:

Column 1: Telescope used in the observation. (see Paper I for the abbreviations).

Column 2: Beam width of the telescope, in arc minutes.

Column 3: Ratio between the HI and optical isophotal diameters, $D_{\mathrm{HI}} / D_{0}$.

Column 4: References. The reference numbers are the same as those of the catalogue of Paper I.

\section{Distance discussion}

The distance may be worth a special consideration, due to the role that it plays in Figs. 2 and 3. Some galaxies have relatively well-known distance, as NGC 224, NGC 598, IC 1613, WLM, Sext A of Local Group, or NGC 3109. For galaxies belonging to the M 81 group (NGC 2366, NGC 2403, NGC 3034, NGC 3077, NGC 4236, IC 2574 and HoII), the adopted distance is $3.25 \mathrm{Mpc}$. The Canes Venatici Group (NGC 4144, NGC 4258, NGC 4395, NGC 4449, NGC 4736, DDO 125) seems to be located between 4.5 and $7 \mathrm{Mpc}$, although the more generally accepted distance is $6.6 \mathrm{Mpc}$ (adopted here). The galaxy belonging to the NGC 5128 group (NGC 5236) has an adopted distance of 4 Mpc. The M101 group (NGC 5055, NGC 5457 and NGC 5474) is located between 7 and $7.2 \mathrm{Mpc}$ (the distance adopted here is $7 \mathrm{Mpc}$ ). For the Circinus galaxy, we adopt the value given by Freeman et al. (1977). The distance given in the literature for NGC 1560 varies between 2.9 and $3.7 \mathrm{Mpc}$; we adopt an intermediate value of $3.3 \mathrm{Mpc}$. For galaxies in the Virgo Cluster, even with low velocities, we adopt a distance of $16.8 \mathrm{Mpc}$. Sculptor group galaxies have been located by different authors between 1.6 and $3.5 \mathrm{Mpc}$ (Huchtmeier \& Seiradakis 1985; Carignan \& Puche 1990; Puche et al. 1991; Hummel et al. 1986). Because of the contrast between nearest and furthest positions assumed for the galaxies of this Group (NGC 55 and NGC 247), we do not adopt any distance, but we kept the lower and higher values to estimate the parameters that depend on distance. This issue also happens for the galaxies: NGC 6946, DDO 154, IC 10, A0355+66, IC 342 and DDO 69 (Leo A). The remaining galaxies have velocities (referred to the Local Group) greater than $400 \mathrm{~km} \mathrm{~s}^{-1}$, therefore we calculate their distances with the Hubble constant $\left(H_{0}=75 \mathrm{Mpc} / \mathrm{km} \mathrm{s}^{-1}\right)$.

\section{General discussion}

For the following discussion, we consider the sample of 116 galaxies listed in Table 1 . The inhomogeneity is evi- dent, since the data were extracted from observations with different radio telescopes. We ought to be careful particularly with the interferometric observations, because the extended component of the gas may have diminished values. Unless it has been added, the missing short spacing causes a zero-level depression, and large-scale structures may be lost. We can see this effect by comparing the values of the relative $\mathrm{HI}$ extension for the galaxies NGC 55, NGC 691, NGC 2366 and DDO 154 (see Table 1): the gas extension for interferometric observations is smaller than for single-dish observations, and the reduction is not larger than about $30 \%$. Nevertheless, the interferometric observations of NGC 4236 seem to have detected the largest extension of gas.

Furthermore, if we compare the values of the gas extension among different single-dish observations of the same galaxy, we also find uncertainties. The errors, at most, reach values of $10 \%-15 \%$ (see NGC 4278 , NGC 5194 and IC 10 in Table 1).

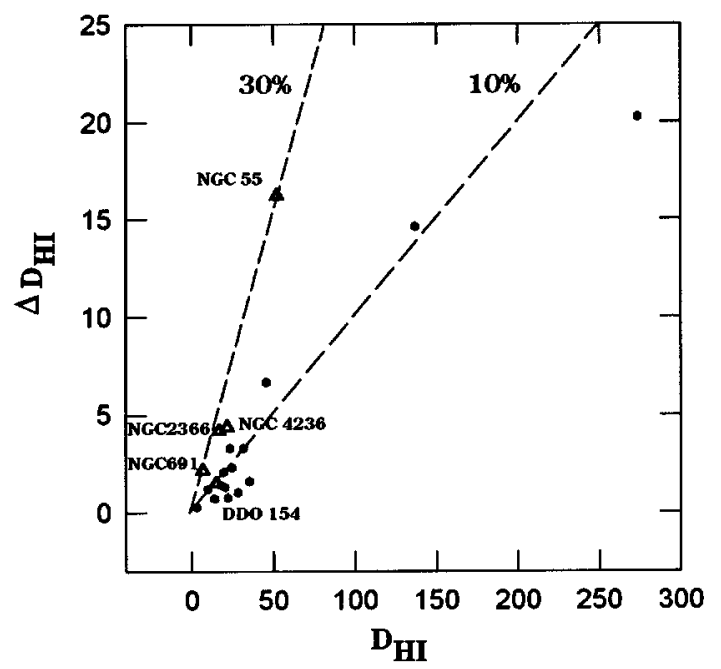

Fig. 1. Maximum difference in the calculated HI extension derived from different observations for a given galaxy, $\Delta D_{\mathrm{HI}}$, in arcmin, versus the mean value of the $\mathrm{HI}$ extension, $D_{\mathrm{HI}}$, in arcmin. Hollow diamond symbols are for differences between single dish and interferometrical data. Filled dots are for differences between single-dish data. The slopes of the lines represent errors of $10 \%$ and $30 \%$

We plot in Fig. 1 the differences of values for the HI isophotal diameter between single dish-single dish and single dish-interferometric observations, as discussed previously. The lines whose slopes represent errors of $10 \%$ and $30 \%$ have also been drawn in the figure. These differences come from errors produced as much in the observations and the HI maps as in the determinations of the isophotal HI diameters. These errors include the disagreements produced by the observations with different radio telescopes.

Figures 2, 4 and 5, show two lines that represent errors of $10 \%$ and $30 \%$ in the relative gas extension. It turns out 
Table 1. Optical and HI parameters for the sample of galaxies used in this analysis

\begin{tabular}{|c|c|c|c|c|c|c|c|c|c|}
\hline$(1)$ & $(2)$ & $(3)$ & $(4)$ & $(5)$ & (6) & $(7)$ & $(8)$ & (9) & $(10)$ \\
\hline Galaxy & Alt.Name & $D_{0}$ & Type & $i$ & Distance & $A(0)$ & $M_{\mathrm{HI}}$ & $\rho_{\mathrm{HI}}$ & $\sigma_{\mathrm{HI}}$ \\
\hline Telescope & HPBW & $D_{\mathrm{HI}} / D_{0}$ & Ref. & & & & & & \\
\hline NGC 7819 & UGC 26 & 1.60 & SB3 & 42 & 69.7 & 32.4 & 11.66 & 1.40 & 2.08 \\
\hline VLA & $1.17 \times 1.0$ & 2.6 & 392 & & & & & & \\
\hline NGC 55 & ESO 293-50 & 32.50 & SB9 & 90 & $1.6-3.5$ & $15.1-33.1$ & $1.69-8.0$ & 90.94 & 3.66 \\
\hline Parkes & 13.5 & 1.6 & 325 & & & & & & \\
\hline VLA & $0.83 \times 0.58$ & 1.1 & 219 & & & & & & \\
\hline VLA & 0.75 & 1.2 & 308 & & & & & & \\
\hline VLA & 1.08 & 1.2 & 68 & & & & & & \\
\hline IC 10 & UGC 192 & 13.40 & IBM10 & 40 & $1.0-3.0$ & $3.9-11.7$ & $0.27-2.43$ & 2.25 & 2.20 \\
\hline MkIA & 12.0 & 3.4 & 104 & & & & & & \\
\hline Effelsb & $8.3 \times 8.7$ & 2.9 & 207 & & & & & & \\
\hline NGC 224 & M 31 & 202.80 & S3 & 78 & 0.69 & 40.8 & 3.77 & 0.29 & 1.58 \\
\hline DRAO & 36.0 & 1.3 & 18 & & & & & & \\
\hline Effelsb & 8.8 & 1.4 & 114 & & & & & & \\
\hline NGC 247 & ESO 540-22 & 21.90 & SB7 & 74 & $1.6-3.5$ & $10.2-22.3$ & $0.52-2.49$ & 0.63 & 2.82 \\
\hline VLA & 1.08 & 1.5 & 68 & & & & & & \\
\hline NGC 262 & Mrk 348 & 1.13 & S0 & 9 & 63.5 & 20.9 & 15.77 & 4.59 & 0.57 \\
\hline Arecibo & 3.6 & 9.0 & 285 & & & & & & \\
\hline IC 1613 & DDO 8 & 16.48 & IB10 & 30 & 0.8 & 3.8 & 0.08 & 0.71 & 1.53 \\
\hline $91 \mathrm{~m}$ & 10.84 & 2.2 & 339 & & & & & & \\
\hline Effelsb & $8.5 / 9.0$ & 2.1 & 216 & & & & & & \\
\hline Zw502-4 & & 0.45 & $\mathrm{~S} 2$ & 41 & 73.2 & 9.6 & 1.19 & 1.65 & 0.85 \\
\hline VLA & $1.17 \times 1.0$ & 4.4 & 392 & & & & & & \\
\hline NGC 598 & M 33 & 73.28 & S6 & 55 & 0.69 & 14.7 & 1.45 & 0.85 & 2.36 \\
\hline $\mathrm{MkI}$ & $14.0 \times 18.0$ & 1.7 & 122 & & & & & & \\
\hline $91 \mathrm{~m}$ & $11.4 \times 10.0$ & 1.9 & 154 & & & & & & \\
\hline Nancay & $4.0 \times 24.0$ & 1.9 & 203 & & & & & & \\
\hline NGC 628 & M 74 & 10.67 & S5 & 24 & 11.2 & 34.8 & 13.91 & 1.46 & 1.93 \\
\hline $91 \mathrm{~m}$ & 10.84 & 2.8 & 339 & & & & & & \\
\hline Arecibo & 3.9 & 2.7 & 71 & & & & & & \\
\hline $\mathrm{A} 0137+15$ & DDO 13 & 4.79 & $\mathrm{I} 10$ & 42 & 8.4 & 11.6 & 0.50 & 0.46 & 1.81 \\
\hline VLA & $0.95 \times 0.83$ & 1.6 & 72 & & & & & & \\
\hline NGC 660 & UGC 1201 & 8.55 & SB1 & 79 & 13.6 & 33.8 & 7.03 & 0.78 & 1.95 \\
\hline $91 \mathrm{~m}$ & 10.84 & 2.0 & 339 & & & & & & \\
\hline UGC 1297 & UM 372 & 1.10 & I10 & 45 & 24.5 & 7.8 & 0.66 & 1.36 & 3.09 \\
\hline VLA & $1.07 \times 0.85$ & 2.1 & 395 & & & & & & \\
\hline NGC 691 & UGC 1305 & 3.66 & SR4 & 43 & 38.1 & 40.6 & 4.50 & 0.35 & 0.96 \\
\hline Arecibo & 3.9 & 1.9 & 178 & & & & & & \\
\hline VLA & $0.95 \times 0.92$ & 1.3 & 443 & & & & & & \\
\hline NGC 694 & Mrk 363 & 3.82 & $\mathrm{C}-2$ & 59 & 41.9 & 46.6 & 2.66 & 0.16 & 6.22 \\
\hline VLA & 0.6 & 0.5 & 443 & & & & & & \\
\hline NGC 772 & Arp 78 & 7.53 & SM3 & 57 & 35.2 & 77.1 & 26.37 & 0.56 & 1.41 \\
\hline $91 \mathrm{~m}$ & 10.84 & 2.0 & 339 & & & & & & \\
\hline NGC 784 & UGC 1501 & 6.73 & SB8 & 90 & 5.4 & 10.6 & 0.45 & 0.51 & 1.29 \\
\hline $91 \mathrm{~m}$ & 10.84 & 2.0 & 339 & & & & & & \\
\hline NGC 891 & UGC 1831 & 14.42 & S3 & 90 & 10.1 & 42.4 & 4.08 & 0.29 & 2.00 \\
\hline $91 \mathrm{~m}$ & 10.84 & 1.2 & 339 & & & & & & \\
\hline NGC 925 & UGC 1913 & 11.30 & SB7 & 57 & 10.1 & 33.2 & 9.71 & 1.12 & 5.70 \\
\hline $91 \mathrm{~m}$ & 10.84 & 1.4 & 339 & & & & & & \\
\hline $91 \mathrm{~m}$ & 10.84 & 1.4 & 199 & & & & & & \\
\hline NGC 1055 & UGC 2173 & 7.76 & SB3 & 76 & 14.3 & 32.3 & 5.38 & 0.65 & 6.54 \\
\hline $91 \mathrm{~m}$ & 10.84 & 1.0 & 339 & & & & & & \\
\hline IC 342 & UGC 2847 & 45.19 & SB6 & 13 & $1.5-4.0$ & $19.7-52.6$ & $2.60-18.49$ & 0.85 & 2.62 \\
\hline $91 \mathrm{~m}$ & 10.0 & 1.8 & 338 & & & & & & \\
\hline$A 0355+66$ & UGC A86 & 1.62 & $\mathrm{I} 10$ & 33 & $1.5-4.0$ & $0.7-1.9$ & $0.38-2.67$ & 95.15 & 2.26 \\
\hline $91 \mathrm{~m}$ & 10.0 & 20.5 & 338 & & & & & & \\
\hline NGC 1560 & bfIC 2062 & 11.43 & S7 & 86 & 3.3 & 11.0 & 1.14 & 1.20 & 2.27 \\
\hline $91 \mathrm{~m}$ & 10.84 & 2.3 & 339 & & & & & & \\
\hline
\end{tabular}


Table 1. continued

\begin{tabular}{|c|c|c|c|c|c|c|c|c|c|}
\hline$(1)$ & $(2)$ & $(3)$ & $(4)$ & $(5)$ & $(6)$ & $(7)$ & $(8)$ & $(9)$ & $(10)$ \\
\hline Galaxy & Alt.Name & $D_{0}$ & Type & $i$ & Distance & $A(0)$ & $M_{\mathrm{HI}}$ & $\rho_{\mathrm{HI}}$ & $\sigma_{\mathrm{HI}}$ \\
\hline Telescope & HPBW & $D_{\mathrm{HI}} / D_{0}$ & Ref. & & & & & & \\
\hline NGC 1792 & ESO 305-0 & 5.27 & SB4 & 63 & 16.2 & 24.8 & 2.68 & 0.55 & 6.79 \\
\hline VLA & $0.67 \times 0.55$ & 0.9 & 116 & & & & & & \\
\hline NGC 2146 & UGC 3429 & 6.46 & SBM2 & 59 & 14.8 & 27.8 & 8.98 & 1.47 & 1.08 \\
\hline $91 \mathrm{~m}$ & $10.2 \times 11.3$ & 3.7 & 141 & & & & & & \\
\hline NGC 2146A & UGC 3439 & 3.30 & SBM5 & 71 & 14.8 & 14.2 & 1.69 & 1.06 & 3.28 \\
\hline $91 \mathrm{~m}$ & $10.2 \times 11.3$ & 1.8 & 141 & & & & & & \\
\hline NGC 2336 & UGC 3809 & 7.35 & SBR4 & 59 & 32.2 & 68.9 & 18.49 & 0.49 & 1.23 \\
\hline $91 \mathrm{~m}$ & 10.92 & 2.0 & 339 & & & & & & \\
\hline NGC 2366 & DDO 42 & 8.40 & IB10 & 90 & 3.25 & 7.9 & 0.72 & 1.46 & 3.01 \\
\hline $91 \mathrm{~m}$ & 10.84 & 2.2 & 339 & & & & & & \\
\hline VLA & 1.08 & 1.5 & 68 & & & & & & \\
\hline NGC 2403 & UGC 3918 & 22.80 & SB6 & 57 & 3.25 & 21.6 & 4.11 & 1.12 & 3.46 \\
\hline $91 \mathrm{~m}$ & 10.84 & 1.8 & 339 & & & & & & \\
\hline NGC 2541 & UGC 4284 & 6.62 & SB6 & 62 & 8.4 & 16.2 & 2.73 & 1.32 & 2.73 \\
\hline $91 \mathrm{~m}$ & 10.84 & 2.2 & 339 & & & & & & \\
\hline $\mathrm{A} 0813+70$ & HoII & 8.09 & $\mathrm{C} 10$ & 43 & 3.25 & 7.7 & 0.94 & 2.04 & 2.69 \\
\hline Effelsb & $8.5 / 9.0$ & 2.7 & 216 & & & & & & \\
\hline $91 \mathrm{~m}$ & 10.84 & 2.8 & 339 & & & & & & \\
\hline NGC 2655 & Arp 225 & 4.90 & SB0 & 36 & 21.4 & 30.5 & 3.66 & 0.50 & 0.55 \\
\hline Effelsb & 9.0 & 3.0 & 212 & & & & & & \\
\hline NGC 2712 & UGC 4708 & 2.90 & SB3 & 60 & 24.8 & 21.0 & 4.00 & 1.16 & 0.72 \\
\hline Effelsb & 9.0 & 4.0 & 213 & & & & & & \\
\hline NGC 2715 & UGC 4759 & 4.97 & SB5 & 74 & 20.3 & 29.3 & 16.81 & 2.47 & 1.07 \\
\hline Effelsb & 9.0 & 4.8 & 212 & & & & & & \\
\hline NGC 2777 & UGC 4823 & 0.87 & $\mathrm{~S} 2$ & 48 & 17.8 & 4.5 & 0.63 & 3.96 & 1.31 \\
\hline Arecibo & 3.9 & 5.5 & 178 & & & & & & \\
\hline NGC 2841 & UGC 4966 & 8.05 & $\mathrm{~S} 3$ & 69 & 9.5 & 22.2 & 3.61 & 0.92 & 0.75 \\
\hline $91 \mathrm{~m}$ & 10.84 & 3.5 & 339 & & & & & & \\
\hline $\mathrm{A} 0930+55 \mathrm{~A}$ & IZw18 & 0.30 & CM5 & 11 & 1.3 & 1.0 & 0.09 & 11.65 & 4.15 \\
\hline WSRT & $0.45 \times 0.54$ & 5.3 & 257 & & & & & & \\
\hline $\mathbf{A 0 9 5 6}+30$ & LeoA & 5.20 & IB10 & 61 & $0.5-1.5$ & $0.8-2.3$ & $0.004-0.04$ & 0.87 & 1.39 \\
\hline $\mathrm{HM}-\mathrm{C}$ & $1.5 \times 3.0$ & 2.5 & 7 & & & & & & \\
\hline NGC 3073 & Mrk 131 & 1.27 & LB-3 & 25 & 17.5 & 6.5 & 0.27 & 0.83 & 0.55 \\
\hline VLA & $1.11 \times 0.95$ & 3.9 & 224 & & & & & & \\
\hline MCG 9-17-9 & & 0.77 & & 80 & 54.0 & 12.1 & 2.62 & 2.27 & 2.53 \\
\hline VLA & $1.11 \times 0.95$ & 3.0 & 224 & & & & & & \\
\hline NGC 3077 & UGC 5398 & 5.56 & S0 & 37 & 3.25 & 5.3 & 1.00 & 4.59 & 0.79 \\
\hline MkIA & 12.0 & 7.6 & 13 & & & & & & \\
\hline NGC 3109 & DDO 236 & 19.77 & SB9 & 90 & 2.2 & 12.7 & 1.80 & 1.43 & 2.70 \\
\hline Parkes & 13.5 & 2.3 & 412 & & & & & & \\
\hline Effelsb & 9.0 & 2.3 & 215 & & & & & & \\
\hline A1008-04 & SextansA & 5.92 & IB10 & 38 & 1.4 & 52.5 & 0.13 & 2.67 & 1.85 \\
\hline Effelsb & $8.5 / 9.0$ & 3.8 & 216 & & & & & & \\
\hline NGC 3187 & Arp 316 & 3.01 & SBM5 & 68 & 21.1 & 18.5 & 1.12 & 0.42 & 1.85 \\
\hline VLA & $1.02 \times 0.99$ & 1.5 & 464 & & & & & & \\
\hline NGC 3190 & Arp 316 & 4.43 & SM1 & 82 & 16.6 & 21.4 & 0.34 & 0.09 & 1.15 \\
\hline VLA & $1.02 \times 0.99$ & 0.9 & 464 & & & & & & \\
\hline NGC 3198 & UGC 5572 & 8.55 & SBR5 & 70 & 9.4 & 23.4 & 5.30 & 1.23 & 1.82 \\
\hline $91 \mathrm{~m}$ & 10.84 & 2.6 & 339 & & & & & & \\
\hline IC 2574 & DDO 81 & 13.34 & CB9 & 90 & 3.25 & 12.6 & 1.03 & 0.82 & 3.41 \\
\hline MkIA & 12.0 & 1.5 & 13 & & & & & & \\
\hline $91 \mathrm{~m}$ & 10.84 & 1.6 & 339 & & & & & & \\
\hline NGC 3338 & UGC 5826 & 6.00 & S5 & 54 & 15.7 & 27.4 & 7.35 & 1.24 & 1.98 \\
\hline $91 \mathrm{~m}$ & 10.84 & 2.5 & 339 & & & & & & \\
\hline NGC 3344 & UGC 5840 & 7.10 & SBR4 & 24 & 6.9 & 14.3 & 2.10 & 1.31 & 1.73 \\
\hline $91 \mathrm{~m}$ & 10.84 & 2.9 & 339 & & & & & & \\
\hline Arecibo & 3.9 & 2.6 & 109 & & & & & & \\
\hline
\end{tabular}


Table 1. continued

\begin{tabular}{|c|c|c|c|c|c|c|c|c|c|}
\hline (1) & $(2)$ & $(3)$ & $(4)$ & $(5)$ & $(6)$ & (7) & $(8)$ & (9) & $(10)$ \\
\hline Galaxy & Alt.Name & $D_{0}$ & Type & $i$ & Distance & $A(0)$ & $M_{\mathrm{HI}}$ & $\rho_{\mathrm{HI}}$ & $\sigma_{\mathrm{HI}}$ \\
\hline Telescope & HPBW & $D_{\mathrm{HI}} / D_{0}$ & Ref. & & & & & & \\
\hline NGC 3351 & M 95 & 7.50 & SBR3 & 50 & 8.6 & 18.8 & 1.06 & 0.38 & 1.69 \\
\hline Arecibo & 3.9 & 1.5 & 359 & & & & & & \\
\hline NGC 3359 & UGC 5873 & 7.26 & SB5 & 55 & 15.3 & 32.3 & 11.28 & 1.37 & 2.38 \\
\hline $91 \mathrm{~m}$ & 10.84 & 2.4 & 339 & & & & & & \\
\hline NGC 3368 & M 96 & 7.64 & SBR2 & 49 & 10.2 & 22.7 & 2.33 & 0.57 & 1.68 \\
\hline $91 \mathrm{~m}$ & 10.84 & 1.8 & 339 & & & & & & \\
\hline Arecibo & 3.9 & 1.9 & 359 & & & & & & \\
\hline NGC 3389 & UGC 5914 & 2.77 & S5 & 63 & 15.6 & 12.6 & 1.58 & 1.27 & 3.17 \\
\hline Arecibo & 3.9 & 2.0 & 359 & & & & & & \\
\hline NGC 3512 & UGC 6128 & 1.61 & SB5 & 23 & 17.7 & 8.3 & 0.72 & 1.33 & 1.58 \\
\hline WSRT & 1.0 & 2.9 & 441 & & & & & & \\
\hline NGC 3521 & UGC 6150 & 11.02 & SB4 & 65 & 8.2 & 26.3 & 4.87 & 0.89 & 2.48 \\
\hline $91 \mathrm{~m}$ & 10.84 & 1.9 & 339 & & & & & & \\
\hline NGC 3623 & M 65 & 9.73 & SBM1 & 90 & 9.1 & 25.8 & 0.32 & 0.06 & 2.48 \\
\hline Arecibo & 3.3 & 0.5 & 180 & & & & & & \\
\hline NGC 3627 & M 66 & 9.23 & SBM3 & 67 & 8.0 & 21.5 & 0.60 & 0.16 & 1.35 \\
\hline Arecibo & 3.3 & 1.1 & 180 & & & & & & \\
\hline NGC 3718 & Arp 214 & 8.05 & SBM1 & 68 & 14.4 & 33.7 & 7.40 & 0.83 & 1.87 \\
\hline $\mathrm{HM}-\mathrm{C}$ & $3.7 \times 4.6$ & 2.1 & 8 & & & & & & \\
\hline UM 452 & & 0.83 & L-2 & 82 & 15.9 & 3.8 & 0.05 & 0.43 & 0.48 \\
\hline VLA & $1.53 \times 1.45$ & 3.0 & 395 & & & & & & \\
\hline IC 745 & UM 465 & 0.79 & L-2 & 25 & 11.6 & 2.7 & 0.03 & 0.48 & 0.39 \\
\hline VLA & $1.03 \times 0.88$ & 3.5 & 395 & & & & & & \\
\hline UGC 6917 & & 3.53 & DB9 & 64 & 13.2 & 13.6 & 1.17 & 0.81 & 0.48 \\
\hline MkIA & 12.0 & 4.1 & 12 & & & & & & \\
\hline NGC 3992 & M 109 & 7.67 & SB4 & 54 & 15.2 & 33.9 & 4.37 & 0.48 & 1.20 \\
\hline $91 \mathrm{~m}$ & 10.84 & 2.0 & 339 & & & & & & \\
\hline UGC 6940 & & 0.99 & S5 & 90 & 16.4 & 4.7 & 0.28 & 1.59 & 6.21 \\
\hline VLA & $0.44 \times 0.33$ & 1.6 & 158 & & & & & & \\
\hline NGC 4016 & UGC 6954 & 1.53 & SB8 & 71 & 45.5 & 20.3 & 3.73 & 1.15 & 2.61 \\
\hline WSRT & $0.75 \times 1.0$ & 2.1 & 442 & & & & & & \\
\hline $\mathbf{A} 1156+53$ & UGC 6969 & 1.63 & IB10 & 90 & 16.0 & 7.6 & 0.41 & 0.90 & 4.01 \\
\hline VLA & $0.44 \times 0.33$ & 1.5 & 158 & & & & & & \\
\hline NGC 4123 & UM 477 & 4.38 & SB5 & 43 & 15.6 & 19.9 & 4.06 & 1.30 & 1.66 \\
\hline VLA & $1.07 \times 0.85$ & 2.8 & 395 & & & & & & \\
\hline NGC 4144 & UGC 7151 & 6.05 & SB6 & 83 & 6.6 & 11.6 & 0.52 & 0.49 & 2.51 \\
\hline $\mathrm{HM}-\mathrm{C}$ & $3.7 \times 5.1$ & 1.4 & 10 & & & & & & \\
\hline NGC 4178 & bfIC 3042 & 5.07 & SB8 & 90 & 16.8 & 24.8 & 4.56 & 0.94 & 6.53 \\
\hline VLA & 0.65 & 1.2 & 98 & & & & & & \\
\hline NGC 4222 & UGC 7291 & 3.33 & S7 & 90 & 16.8 & 16.3 & 0.87 & 0.42 & 1.86 \\
\hline VLA & $0.8 \times 0.78$ & 1.5 & 98 & & & & & & \\
\hline NGC 4236 & UGC 7306 & 21.93 & SB8 & 90 & 3.25 & 20.7 & 1.52 & 0.45 & 4.49 \\
\hline $91 \mathrm{~m}$ & 10.84 & 0.9 & 339 & & & & & & \\
\hline VLA & 1.08 & 1.1 & 68 & & & & & & \\
\hline NGC 4237 & UGC 7315 & 2.10 & SBR4 & 52 & 16.8 & 10.3 & 0.34 & 0.41 & 2.83 \\
\hline VLA & $0.9 \times 0.73$ & 1.2 & 98 & & & & & & \\
\hline NGC 4244 & UGC 7322 & 16.56 & S6 & 90 & 6.6 & 31.8 & 5.90 & 0.74 & 4.38 \\
\hline VLA & 1.08 & 1.3 & 68 & & & & & & \\
\hline NGC 4258 & M 106 & 18.66 & SB4 & 71 & 6.6 & 35.8 & 5.01 & 0.49 & 1.71 \\
\hline $91 \mathrm{~m}$ & 10.84 & 1.7 & 339 & & & & & & \\
\hline NGC 4262 & UGC 7365 & 1.88 & LB-3 & 29 & 16.8 & 9.2 & 0.45 & 0.68 & 0.38 \\
\hline Arecibo & $4.14 / 5.0$ & 4.2 & 84 & & & & & & \\
\hline NGC 4278 & UGC 7386 & 4.12 & E-5 & 29 & 13.0 & 15.6 & 0.46 & 0.24 & 0.45 \\
\hline Arecibo & $4.14 / 5.0$ & 2.4 & 84 & & & & & & \\
\hline Arecibo & 3.5 & 2.2 & 236 & & & & & & \\
\hline NGC 4385 & UM 499 & 2.06 & LB-1 & 58 & 26.5 & 15.9 & 0.98 & 0.49 & 1.71 \\
\hline VLA & $0.98 \times 0.9$ & 1.7 & 395 & & & & & & \\
\hline
\end{tabular}


Table 1. continued

\begin{tabular}{|c|c|c|c|c|c|c|c|c|c|}
\hline$(1)$ & $(2)$ & $(3)$ & $(4)$ & $(5)$ & $(6)$ & (7) & $(8)$ & (9) & $(10)$ \\
\hline Galaxy & Alt.Name & $D_{0}$ & Type & $i$ & Distance & $A(0)$ & $M_{\mathrm{HI}}$ & $\rho_{\mathrm{HI}}$ & $\sigma_{\mathrm{HI}}$ \\
\hline Telescope & HPBW & $D_{\mathrm{HI}} / D_{0}$ & Ref. & & & & & & \\
\hline NGC 4395 & UGC 7524 & 13.10 & SBR9 & 38 & 6.6 & 25.2 & 3.40 & 0.68 & 1.70 \\
\hline $91 \mathrm{~m}$ & 10.84 & 2.0 & 339 & & & & & & \\
\hline UGC 7531 & UM 500 & 1.07 & IBM10 & 35 & 23.7 & 7.4 & 0.39 & 0.92 & 1.73 \\
\hline VLA & $1.03 \times 0.95$ & 2.3 & 395 & & & & & & \\
\hline IC 3365 & UGC 7563 & 2.16 & IB10 & 79 & 16.8 & 10.6 & 0.41 & 0.47 & 5.79 \\
\hline VLA & 0.4 & 0.9 & 382 & & & & & & \\
\hline $\mathrm{A} 1225+43$ & DDO 125 & 4.26 & D10 & 66 & 6.6 & 8.2 & 0.27 & 0.50 & 2.24 \\
\hline WSRT & $0.85 \times 1.22$ & 1.5 & 404 & & & & & & \\
\hline NGC 4449 & UGC 7592 & 6.12 & IB10 & 43 & 6.6 & 11.8 & 7.32 & 6.72 & 2.48 \\
\hline Effelsb & 9.3 & 5.2 & 21 & & & & & & \\
\hline IC 3522 & DDO 136 & 1.57 & IB10 & 77 & 16.8 & 7.7 & 0.71 & 1.52 & 3.45 \\
\hline VLA & 0.83 & 2.2 & 381 & & & & & & \\
\hline VLA & 0.4 & 2.0 & 382 & & & & & & \\
\hline NGC 4565 & UGC 7772 & 16.00 & S3 & 90 & 13.0 & 60.5 & 11.83 & 0.41 & 1.60 \\
\hline $91 \mathrm{~m}$ & 10.84 & 1.6 & 339 & & & & & & \\
\hline NGC 4569 & M 90 & 9.82 & SBM2 & 68 & 16.8 & 48.0 & 0.82 & 0.05 & 2.82 \\
\hline WSRT & $0.42 \times 0.89$ & 0.4 & 452 & & & & & & \\
\hline NGC 4618 & Arp 23 & 4.14 & SBM9 & 40 & 7.9 & 9.5 & 1.27 & 1.78 & 5.48 \\
\hline WSRT & 0.75 & 1.8 & 442 & & & & & & \\
\hline NGC 4631 & Arp 281 & 15.56 & SBM7 & 50 & 8.2 & 37.1 & 12.78 & 1.18 & 2.94 \\
\hline $91 \mathrm{~m}$ & 10.0 & 2.0 & 320 & & & & & & \\
\hline NGC 4651 & Arp 189 & 4.05 & SM5 & 50 & 16.8 & 19.8 & 4.01 & 1.30 & 2.25 \\
\hline VLA & $1.03 \times 0.98$ & 2.4 & 360 & & & & & & \\
\hline NGC 4656 & UGC 7907 & 15.10 & SBM9 & 90 & 8.6 & 37.8 & 6.66 & 0.59 & 1.64 \\
\hline $91 \mathrm{~m}$ & 10.0 & 1.9 & 320 & & & & & & \\
\hline UGC 7906 & & 1.07 & $\mathrm{I} 10$ & 52 & 12.2 & 3.8 & 0.15 & 1.31 & 1.36 \\
\hline VLA & $0.4 \times 0.4$ & 3.1 & 382 & & & & & & \\
\hline NGC 4689 & UGC 7965 & 4.33 & $\mathrm{~S} 4$ & 36 & 16.8 & 21.2 & 0.76 & 0.22 & 2.16 \\
\hline VLA & $0.78 \times 0.72$ & 1.0 & 98 & & & & & & \\
\hline NGC 4725 & UGC 7989 & 10.84 & SBR2 & 48 & 13.0 & 41.0 & 11.83 & 0.89 & 3.97 \\
\hline Arecibo & 3.9 & 1.5 & 177 & & & & & & \\
\hline NGC 4736 & M 94 & 11.25 & $\mathrm{SR} 2$ & 38 & 6.6 & 21.6 & 0.98 & 0.27 & 1.18 \\
\hline $91 \mathrm{~m}$ & 10.84 & 1.5 & 339 & & & & & & \\
\hline NGC 4747 & Arp 159 & 3.48 & SB6 & 73 & 13.0 & 13.2 & 2.84 & 2.08 & 2.31 \\
\hline Arecibo & 3.9 & 3.0 & 177 & & & & & & \\
\hline NGC 4789A & DDO 154 & 3.06 & BM10 & 50 & $4.0-10.0$ & $3.6-8.9$ & $0.44-2.78$ & 4.45 & 1.78 \\
\hline Arecibo & 3.8 & 5.1 & 245 & & & & & & \\
\hline Arecibo & 3.2 & 4.8 & 198 & & & & & & \\
\hline VLA & 0.75 & 4.6 & 87 & & & & & & \\
\hline NGC 5022 & ESO 576-14 & 2.52 & $\mathrm{~S} 3$ & 90 & 37.3 & 27.4 & 3.34 & 0.57 & 2.52 \\
\hline VLA & $1.24 \times 0.87$ & 1.5 & 234 & & & & & & \\
\hline NGC 5033 & UGC 8307 & 10.69 & S5 & 64 & 12.2 & 38.0 & 6.70 & 0.59 & 3.01 \\
\hline $91 \mathrm{~m}$ & 10.84 & 1.4 & 339 & & & & & & \\
\hline $\mathbf{A} 1313+25$ & DDO 170 & 1.29 & $\mathrm{I} 10$ & 90 & 12.4 & 4.6 & 0.48 & 2.79 & 3.10 \\
\hline VLA & $0.4 \times 0.33$ & 3.0 & 255 & & & & & & \\
\hline NGC 5055 & M 63 & 12.59 & $\mathrm{~S} 4$ & 58 & 7.0 & 25.7 & 6.20 & 1.20 & 1.64 \\
\hline $91 \mathrm{~m}$ & 10.84 & 2.7 & 339 & & & & & & \\
\hline NGC 5194 & M 51 & 11.14 & SM4 & 54 & 7.0 & 22.7 & 3.05 & 0.75 & 1.70 \\
\hline MkIA & 12.0 & 2.2 & 14 & & & & & & \\
\hline $91 \mathrm{~m}$ & $9.0 \times 10.2$ & 1.9 & 323 & & & & & & \\
\hline NGC 5236 & M 83 & 13.3 & SB5 & 27 & 4.0 & 15.5 & 6.15 & 3.26 & 0.85 \\
\hline Effelsb & $8.5 / 9.0$ & 6.2 & 210 & & & & & & \\
\hline NGC 5301 & UGC 8711 & 4.21 & SB4 & 90 & 21.7 & 26.6 & 5.09 & 0.91 & 0.89 \\
\hline Effelsb & 9.0 & 3.2 & 213 & & & & & & \\
\hline
\end{tabular}


Table 1. continued

\begin{tabular}{|c|c|c|c|c|c|c|c|c|c|}
\hline$(1)$ & $(2)$ & $(3)$ & $(4)$ & $(5)$ & $(6)$ & $(7)$ & $(8)$ & $(9)$ & $(10)$ \\
\hline Galaxy & Alt.Name & $D_{0}$ & Type & $i$ & Distance & $A(0)$ & $M_{\mathrm{HI}}$ & $\rho_{\mathrm{HI}}$ & $\sigma_{\mathrm{HI}}$ \\
\hline Telescope & HPBW & $D_{\mathrm{HI}} / D_{0}$ & Ref. & & & & & & \\
\hline NGC 5457 & M 101 & 28.58 & SBM6 & 22 & 7.0 & 58.2 & 21.27 & 0.80 & 1.99 \\
\hline $91 \mathrm{~m}$ & 10.84 & 2.0 & 339 & & & & & & \\
\hline Effelsb & $8.3 \times 8.7$ & 2.0 & 218 & & & & & & \\
\hline NGC 5474 & Arp 26 & 4.81 & SM6 & 27 & 7.0 & 9.8 & 1.37 & 1.81 & 1.19 \\
\hline MkIA & 12.0 & 4.0 & 120 & & & & & & \\
\hline $91 \mathrm{~m}$ & 10.84 & 3.9 & 339 & & & & & & \\
\hline Effelsb & $8.3 \times 8.7$ & 3.7 & 218 & & & & & & \\
\hline A1409-65 & Circinus & 11.35 & S3 & 69 & 4.0 & 13.2 & 7.20 & 5.23 & 1.56 \\
\hline Parkes & 15.0 & 5.8 & 144 & & & & & & \\
\hline A1448+35 & IIZw70 & 0.74 & CM4 & 75 & 17.4 & 3.8 & 0.39 & 3.51 & 4.17 \\
\hline WSRT & $0.85 \times 1.45$ & 2.9 & 31 & & & & & & \\
\hline $\mathrm{A} 1449+35$ & IIZw71 & 0.95 & CM5 & 27 & 17.9 & 4.9 & 0.83 & 4.29 & 5.10 \\
\hline WSRT & $0.85 \times 1.45$ & 2.9 & 31 & & & & & & \\
\hline NGC 5832 & UGC 9649 & 3.75 & SB3 & 56 & 8.9 & 9.7 & 1.13 & 1.52 & 1.24 \\
\hline Effelsb & 9.0 & 3.5 & 213 & & & & & & \\
\hline NGC 5907 & UGC 9801 & 12.50 & S5 & 90 & 11.3 & 41.0 & 7.66 & 0.58 & 1.78 \\
\hline $91 \mathrm{~m}$ & 10.84 & 1.8 & 339 & & & & & & \\
\hline NGC 6503 & UGC 11012 & 7.26 & S6 & 73 & 4.0 & 8.5 & 0.71 & 1.26 & 1.86 \\
\hline $91 \mathrm{~m}$ & 10.84 & 2.6 & 339 & & & & & & \\
\hline ESO 400-12 & Klemola31A & 0.78 & S5 & 70 & 107.9 & 24.4 & 1.16 & 0.25 & 1.46 \\
\hline VLA & 0.33 & 1.3 & 95 & & & & & & \\
\hline NGC 6946 & Arp 29 & 16.60 & SB6 & 32 & $4.5-10.0$ & $21.7-48.3$ & $4.48-22.11$ & 1.20 & 3.33 \\
\hline $91 \mathrm{~m}$ & 10.84 & 1.8 & 339 & & & & & & \\
\hline $91 \mathrm{~m}$ & 10.0 & 2.0 & 155 & & & & & & \\
\hline NGC 7280 & UGC 12035 & 2.16 & LB-1 & 53 & 28.0 & 17.6 & 0.24 & 0.10 & 0.99 \\
\hline WSRT & $1.0 \times 1.5$ & 1.0 & 432 & & & & & & \\
\hline NGC 7331 & UGC 12113 & 11.40 & S3 & 75 & 14.9 & 49.5 & 8.88 & 0.46 & 3.20 \\
\hline $91 \mathrm{~m}$ & 10.84 & 1.2 & 339 & & & & & & \\
\hline NGC 7448 & Arp 13 & 2.75 & S4 & 66 & 32.6 & 26.1 & 7.86 & 1.47 & 1.63 \\
\hline Arecibo & 3.9 & 3.0 & 178 & & & & & & \\
\hline NGC 7640 & UGC 12554 & 11.53 & SB5 & 90 & 8.9 & 29.9 & 6.73 & 0.96 & 2.96 \\
\hline $91 \mathrm{~m}$ & 10.84 & 1.8 & 339 & & & & & & \\
\hline A2359-15 & WLM & 11.78 & IB10 & 90 & 1.6 & 5.5 & 0.18 & 0.75 & 1.69 \\
\hline $91 \mathrm{~m}$ & 11.20 & 2.2 & 339 & & & & & & \\
\hline Effelsb & $8.5 / 9.0$ & 2.0 & 216 & & & & & & \\
\hline
\end{tabular}

that, due to the natural spread of values, the meaning of the graphics is not severely affected by the uncertainty in gas extension. In other words, it seems quite possible that these errors do not essentially modify the results obtained in the present paper by using the whole sample, including interferometrical data. Then, we decided to consider all the data for relying on a large sample of galaxies and a wide range of $\mathrm{HI}$ extensions to analyse the behaviour of the gas extension in galaxies. Of course, we must keep in mind that the values of the relative gas extension might be diminished for those galaxies in Table 1 having only interferometric observations, such as DDO 13, NGC 247, Izw 18, etc.

Figure 2 plots the HI extent relative to the optical ratio, $D_{\mathrm{HI}} / D_{0}$, as a function of the lineal optical diameter $A(0)$ in kpc. Due to the fact that this plot is strongly distance-dependent, the galaxies with uncertain distance have been drawn with diamond symbols, and the two extreme values of $A(0)$ have been joined by a dashed line. The two positions of LeoA may indicate that the more appropriate distance is the largest one, i.e. $1.5 \mathrm{Mpc}$.

Figure 2 shows a clear trend for the smallest galaxies to have higher values of the relative extension of neutral hydrogen. One might think that the lower envelope of this trend is produced by spatial resolution effects. For examining these effects, we have separated the data according to different telescopes. From Table 1, the sample contains 47 interferometric observations with spatial resolutions within $20^{\prime \prime}-2^{\prime}$ (VLA and Westerbork telescopes), 3 interferometric observations with lower spatial resolution (Cambridge Half Mile radio telescope), 18 single dish observations (Arecibo) within $3^{\prime}-4.5,64$ observations within $8^{\prime}-11^{\prime}$ (Effelsberg and 300-ft telescopes), and 12 observations with spatial resolution lower than $11^{\prime}(\mathrm{MkI}$ and 


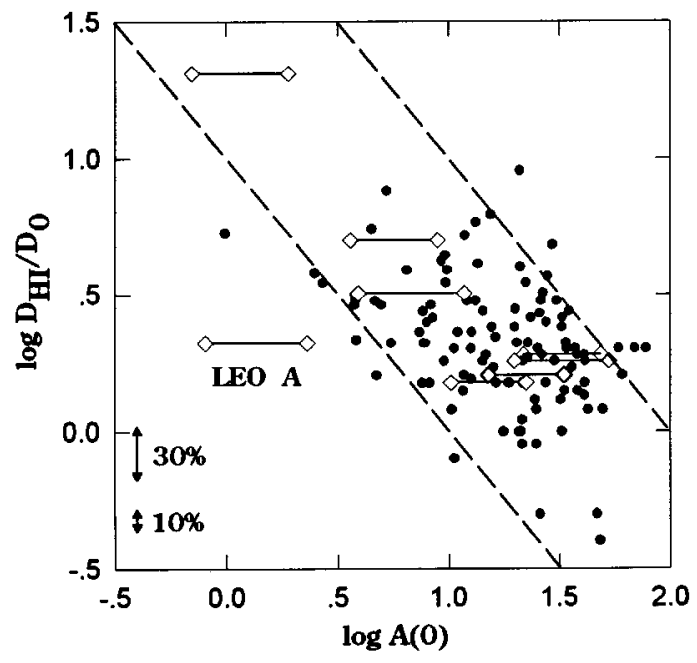

Fig. 2. Ratio between the isophotal $\mathrm{HI}$ and optical extensions, $D_{\mathrm{HI}} / D_{0}$, versus the linear optical diameter, $A(0)$, in Kpc. Diamond symbols joined by a line represent the extreme values of $A(0)$ for galaxies with uncertain distance (see Table 1)

MkIA telescopes, Parkes, Nançay and DRAO). Then, we group the data according to different telescopes and spatial resolutions, as shown in Fig. 3. There are no significant differences in the distribution of the data, with exception of the interferometrical observations. Diamond symbols seem to be spread below those that symbolize single-dish observations. We have also placed in this figure the lines that represent the natural limits of the telescopes calculated with the typical spatial resolution of the data set. The minimum distance taken for calculating these limit lines is $3.25 \mathrm{Mpc}$ (assumed for the M 81 Group), although there are in the sample some nearer galaxies (in the Local Group). As can be seen, the telescope limit for the lowest spatial-resolution data could influence the presence of the lower envelope of the graphic. The rest of the data do not seem to be affected by the spatial resolution. Then we have no doubt about the certainty of the trend in the graphic: the smaller a galaxy, the bigger the gas extension will be found in it. This relation may come from the relation between the gas extension and the angular momentum of a galaxy. We found a similar trend when, instead of $A(0)$, we plotted the angular momentum calculated in first approximation by the product of $A(0)$ and the width of the profile.

On the other hand, it is interesting to note that the apparent lower and upper envelopes that are seen in Figs. 2 and 3 might be produced by a natural range in the sizes of the gas component. In Fig. 2, we traced two lines by hypothesis, that represent gas extensions of 10 and $100 \mathrm{kpc}$. Most of the galaxies seem to be located between these lines. A few galaxies lie outside the lines, having HI extensions smaller or greater than the values quoted previously. But we are dealing with tentative lines, with a parameter (the linear diameter) that depends strongly

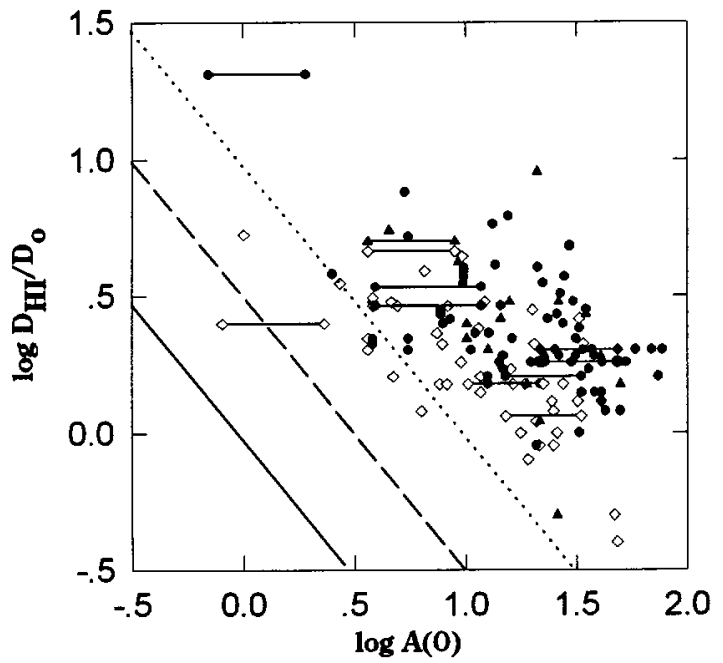

Fig. 3. Same as Fig. 2, where the data were separated according to the telescopes and their spatial resolutions. Hollow diamonds are for interferometrical observations with resolutions between $20^{\prime \prime}$ and $4^{\prime}$, filled triangles are for single-dish (Arecibo) observations with values of HPBW between $3^{\prime}$ and 4.5 and filled dots are for those observations with resolutions lower than $8^{\prime}$. The lines represent the limits of telescopes for the three arrangement of data, calculated with characteristic values of the half power beam resolutions of 1' (full line), 3.5 (dashed line) and $10^{\prime}$ (dotted line)

on the distance and its errors. The best example for the last remark is Leo A, which is the galaxy most detached from the lower established limit, but also has the most uncertain distance.

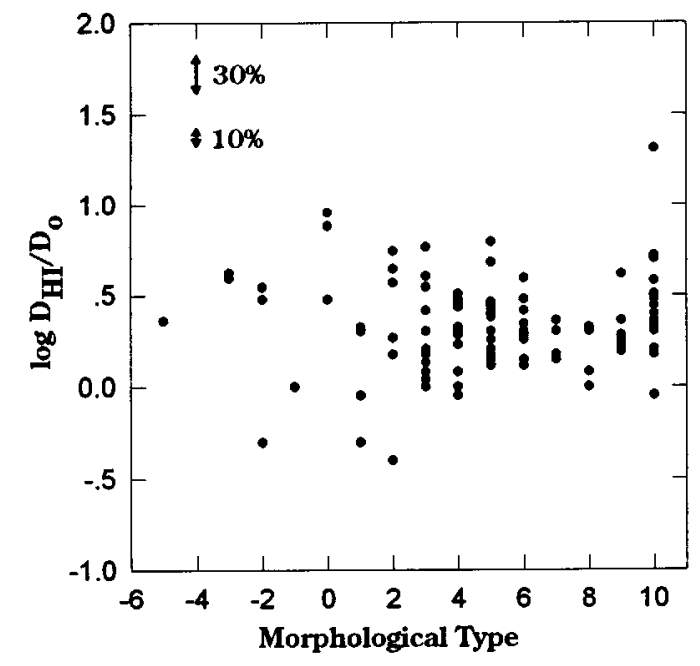

Fig. 4. Ratio $D_{\mathrm{HI}} / D_{0}$ against morphological type

Figure 4 shows the distribution of the ratio $D_{\mathrm{HI}} / D_{0}$ among the different morphological types. Despite the fact that the sample comprises a variety of morphologies, the present data show no dependence of the relative gas 
extension on the type. This result is similar to those obtained by Krumm \& Salpeter (1979), Bosma (1981) and Hewitt et al. (1983). As many early as late-type galaxies have a wide range of extensions of gas. Nevertheless, the values of $D_{\mathrm{HI}} / D_{0}$ for a few galaxies support the usual belief that Irregulars have very large HI envelopes, while the gas in late-type galaxies lies, at most, up to the edge of the optical extension. As can be seen in Fig. 4, three galaxies have HI extensions quite lower than the optical. These are early-type galaxies. Also, the largest relative HI extension belongs to an Irregular galaxy.

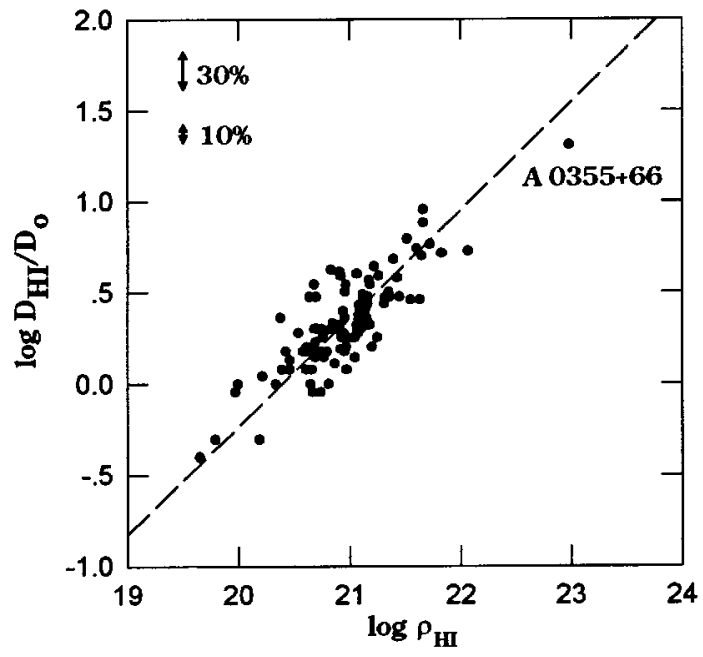

Fig. 5. Variation of $D_{\mathrm{HI}} / D_{0}$ against $\sigma_{\mathrm{HI}}$, the apparent density of HI mass

Figure 5 displays the apparent density of HI, independent of distance, versus the relative gas extension, also distance-independent. The HI density is apparent, because we are supposing that the gas is kept in the optical extension. If the real HI density is approximately the same for all the galaxies, then an increase of the gas extension beyond the optical size means an increase of the gaseous mass, and thus the apparent density. This built-in relationship is displayed in Fig. 5. Despite this dependence being known, we consider it interesting to derive the expression that relates the parameters. So, we can approach the value of the gas extension by means of the apparent HI density, which can be calculated only by an integral profile. A mean least squares fit yields:

$\log \left(D_{\mathrm{HI}} / D_{0}\right)=0.58 \log \left(\rho_{\mathrm{HI}}\right)-11.81$

with a correlation coefficient of 0.85 .

The slope is about 0.5 , which means that there is a correlation of about one to one between the linear size of gas (in kpc) and the HI mass of the galaxy, as found by Hewit et al. (1983). This implies a nearly constant real HI surface density.

It is necessary to mention that we tried to get the truest value of the HI mass. We compared the values of the HI magnitude $\left(m_{21}\right)$ as given by the LEDA catalogue with those obtained by integration of the HI maps as quoted in the catalogue of Paper I. For single-dish telescopes maps, the masses estimated by integration of the HI maps are, in general, greater than those calculated by $m_{21}$ (basically obtained from integral profiles). This is not the case for some galaxies observed by interferometrically. For these galaxies, we adopt the HI masses obtained from $m_{21}$, because of missing of the total $\mathrm{HI}$ gas detection in interferometric observations, such as NGC 3073, UGC 6940, NGC 4618, etc. We found that with this combination of masses, the graphic in Fig. 5 has the least dispersion. Without any doubt, A0355+66 gives an effective weight to this relation, because of its high apparent surface density.

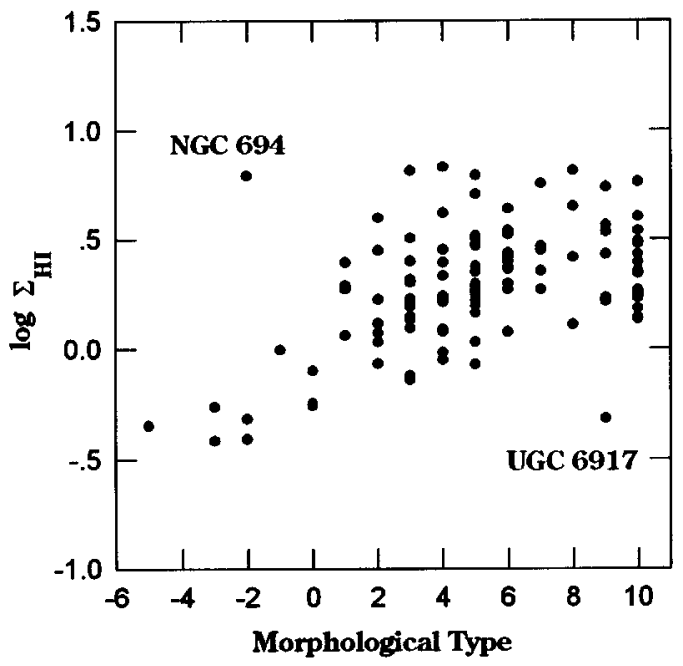

Fig. 6. Real density of HI mass $\left(\Sigma_{\mathrm{HI}}\right)$ versus the morphological type

Figure 6 shows the distribution of the real HI surface density among the morphological types. It appears that Elliptical and S0 galaxies have low surface density of gas, except for NGC 694 as marked in the figure. The NGC 694/IC 167 complex belongs to the NGC 697 group of gravitationally interacting galaxies, with an outstanding HI intergalactic cloud. On this figure, the richest galaxies in HI gas density are the spirals, which have a broad dispersion of values without any remarkable trend. Within the spiral galaxies, the exception is UGC 6917, which is a companion of NGC 4026 and is probably interacting with other galaxies in the vicinity of UGC 6956 and NGC 6922.

In all previous graphics, we found no preferential sector either for isolated (like NGC 628, NGC 2712, NGC 3109, etc.) or interacting galaxies (like NGC 3627, NGC 4631 and NGC 4656, II Zw 70 and II Zw 71), except for those remarked in Fig. 6. Furthermore, we could not find any relationship between the gas extension and other properties of the galaxies, such as surface brightness, colours, infrared and blue magnitudes or profile width. 


\section{Conclusions}

With a large sample of galaxies, we found a propensity in the smaller galaxies to have the larger HI extensions. So the real $\mathrm{HI}$ sizes appear to lie in a defined range.

From the relation between the relative HI diameter and the apparent HI surface density, we give useful expression that predicts roughly how extended a galaxy will be in HI.

We find no dependence of the gas extension on morphological type. Instead, there is a broad dispersion within different types. The galaxies with large HI extensions run from S0 to Irr.

The real surface masses of hydrogen in the sample have values between 0.4 and $6.810^{20}$ at $\mathrm{cm}^{-2}$. There is a broad range of values for Spiral and Irr galaxies, but for Elliptical and S0 galaxies, the real surface masses are generally low.

Acknowledgements. We would like to thank J.C. Olano and J.C. Olalde for reading the paper carefully, J.C. Cersosimo and J.C. Testori for valuable material and discussions, and C. Cristina Miguel and M. Fumagalli for computational support.

\section{References}

Bajaja E., Huchtmeier W.K., Klein U., 1994, A\&A 285, 385

Balkowski C., Bottinelli L., Gouguenheim L, Heidmann J., 1972, A\&A 21, 30

Balkowski C., 1979, A\&A 78, 190

Bosma A., 1981, AJ 86, 1825

Bottinelli L., 1971, A\&A 10, 437

Bottinelli L., Gouguenheim L., 1973, A\&A 29, 425
Briggs F.H., Wolfe A.M., Krumm N., Salpeter E.E., 1980, ApJ 238,510

Carignan C., Puche D., 1990, AJ 100, 641

Fisher J.R., Tully R.B., 1976, A\&A 53, 397

Fouqué P., 1982, Proc. of the workshop "The comparative HI content of normal galaxies" held in Green Bank, West Virginia, p. 46

Fouqué P., 1983, A\&A 122, 273

Freeman K.C., Karlsson B., Lynga G., et al., 1977, A\&A 55, 445

Gallagher J.S., Hunter D.A., 1984, ARA\&A 22, 37

Giovanelli R., Haynes M.P., 1988, Galactic and Extragalactic Radio Astronomy, Verschur G.L. and Kellermann K.I. (eds.) p. 522

Haynes M.P., Giovanelli R., Chincarini G.L., 1984, ARA\&A 22,445

Hewitt J.N., Haynes M.P., Giovanelli R., 1983, AJ 88, 272

Huchtmeier W., 1972, A\&A 17, 207

Huchtmeier W.K., Seiradakis J.H., Materne J., 1981, A\&A 102, 134

Huchtmeier W.K., Seiradakis J.H., 1985, A\&A 143, 216

Hummel E., Dettmar R.J., Wielebinski R., 1986, A\&A 166, 97

Krumm N., Salpeter E.E., 1979, AJ 84, 1138

Krumm N., Salpeter E.E., 1980, AJ 85, 1312

Krumm N., Burstein D., 1984, AJ 89, 1319

Morris M., Wannier P.G., 1980, ApJ 238, L7

Puche D., Carignan C., Wainscoat R.J., 1991, AJ 101, 447

Robinson B.J., van Damme K.J., 1966, Aust. J. Phys. 19, 111

Rots A.H., 1980, A\&AS 41, 189

Sancisi R., 1987, Proc. of the QSO Absorption Lines Meeting, Blades J.C., Turnshek D.A. and Norman C.A. (eds.). Cambridge University Press., p. 241

Schneider S., 1989, ApJ 343, 94 